\title{
Intelligent systems and smart homes
}

\author{
Jong Hyuk Park • Ching-Hsien Hsu • Hamid R. Arabnia
}

Published online: 26 June 2008

(C) Springer Science + Business Media, LLC 2008

The concept of ambient intelligence propagates a vision of smart homes where people are supported and assisted in their daily activities by information technology that is very different from the computer as we know today. In recent years, significant advances have been achieved in the enabling technologies for smart homes, such as the increasingly mature networked appliances, pervasive sensor technologies and various kinds of wired and wireless communication protocols. These advances play a major role in the facilitation of smart homes.

Though, there is not a general agreement on the definition of Home intelligence, many consider Home intelligent to generally be the combination of home network service, home automation service, and internet service to devices, services, as well as offices (in and/or outside home). Recently, home intelligent has been extended to include context aware and situation aware automation by using ubiquitous technologies. For example, in smart home environments, users are progressively offered more personalized services, ranging from home automation, security, monitoring, entertainment, to healthcare. In particular, many research efforts have been targeted to design and

\footnotetext{
J. H. Park $(\bowtie)$

Department of Computer Science and Engineering,

Kyungnam University,

Kyungnam, Korea

e-mail: parkjonghyuk1@hotmail.com

C.-H. Hsu

Department of Computer Science and Information Engineering, Chung Hua University,

Hsinchu, Taiwan

H. R. Arabnia

Computer Science, University of Georgia,

Athens, GA, USA
}

development of smart homes for ageing and disabled people.

It is widely acknowledged that an important step in ubiquitous computing is context-awareness. Services in pervasive and mobile environments need to be contextaware so that they can adapt themselves to rapidly changing situations. Even though many intelligent systems have been built in recent years, there is a still a lack of a general model to guide the system design. The lack of a general model has resulted the construction of systems that suffer from many interoperability issues.

In this context, we envision the creation of smart environments that integrate information, communication and sensing technologies into everyday objects. This special issue is a collection of novel ideas and state-ofthe-art research results on integrating intelligent systems and smart homes. We hope that this special issue will serve as a landmark source for education, information, and reference to students, professionals, and researchers interested in updating their knowledge about intelligent systems and smart homes.

Submissions to this special issue come from the open Call for Papers as well as from the selected papers presented at the 2007 International Workshop on Intelligent Systems and Smart Homes (WISH-07) organized at Niagara Falls, Canada, from August 28th to September 1 st, 2007.

In the paper "Service Integration with UPnP Agent for a Ubiquitous Home Environment", Wally Chen et al. proposed an approach for UPnP devices connecting to outer networks via UPnP aware gateway. They also developed several service oriented applications in a smart campus based on the proposed framework.

The paper "A Novel Memory Management Scheme for Residential Gateways" by Ibrahim Kamel and Beizhong 
Chen presents two algorithms for service replacement and memory management in home gateways.

In the paper "Location-aware Communications in Smart Environments", Ichiro Satoh presents a location-aware communication approach for smart home environments that is based on a symbolic location model that represents the containment relationships between physical objects, computing devices, and places.

The paper "T-LEACH: The Method of Threshold-based Cluster Head Replacement for Wireless Sensor Networks" by Jiman Hong et al. present T-LEACH, which is a threshold-based cluster head replacement scheme for clustering protocols of wireless sensor networks. T-LEACH minimizes the number of cluster head selection by using threshold of residual energy.

In the paper "A Multi-functional Platform for Implementing Intelligent and Ubiquitous Functions of Smart Substations under SCADA", Hak-Man Kim et al. propose a multifunctional platform to implement smart substations effectively. A prototype hardware, functions and communication interfaces on an embedded platform are introduced.

The paper "A Ubiquitous smart home for elderly" by M. W. Raad and Laurence T. Yang presents an approach which develops a cost-effective user-friendly telehealth system to serve the elderly and disabled people in the community. The research also aims at utilizing the state-of-the-art advances in medical instrumentation technology to establish a continuous communication link between patients and caregivers and allow physicians to offer help when needed.

In the paper "Monitoring User Activities in Smart Home Environments", Sajid Hussain et al. propose an architecture and design of a web application for a sensor network monitoring. Further, the variation in received signal strength indicator (RSSI) values is used for knowledge extraction. Experiments are conducted in an indoor room environment to determine the activities of a person.

In the paper "Learning Fuzzy Concept Hierarchy and Measurement with Node Labeling" by Been-Chian Chien et al., the fuzzy characteristics of human knowledge are studied and employed to represent concepts and hierarchical relationships among the concepts. An agglomerative clustering scheme is proposed to learn hierarchical fuzzy concepts from databases.

Finally, we strongly believe that the selected papers make a significant contribution to researchers, practitioners, and students working in the areas of the intelligent systems and smart homes. We would like to express our sincere appreciation to all the authors for their valuable contributions and also to the referees for their cooperation and hard work in reviewing the papers in a timely and professional manner. Our special thanks go to the editorial board for this SI and Profs. R. Ramesh and H. Raghav Rao, who is Editor in Chief of for his supports throughout the whole publication processes.

Jong Hyuk Park received his Ph.D. degree in the Graduate School of Information Security from Korea University, Korea. He is now a professor at the Department of Computer Science and Engineering, Kyungnam University, Korea. He has published many research papers in international journals and conferences. He has been served as chairs, program committee, or organizing committee chair for many international conferences and workshops. He is editor-in-chief of the International Journal of Multimedia and Ubiquitous Engineering (IJMUE), the managing editor of the International Journal of Smart Home (IJSH). In addition, he has served as a guest editor for international journals by some publishers. His research interests include digital forensics, security, ubiquitous and pervasive computing, context awareness, multimedia services, etc. He got the best paper award in IEEE ISA-08 conference, April, 2008.

Ching-Hsien Hsu received the B.S. and Ph.D. degrees in Computer Science from Tung Hai University and Feng Chia University, Taiwan, in 1995 and 1999, respectively. He is currently a faculty of the department of Computer Science and Information Engineering at Chung Hua University, Taiwan. Dr. Hsu has edited more than 10 international journal special issues as a guest editor and has severed many international conferences as various chairs and committee members. Dr. Hsu has published more than 80 academic papers in journals, books, and conference proceedings. He was awarded as one of the annual outstanding researchers by Chung Hua University in 2005, 2006, and 2007 and got the excellent research award in 2008. His research interests include parallel and distributed computing, grid and pervasive computing, intelligent computing, P2P computing, RFID, and smart homes.

Hamid R. Arabnia received a Ph.D. degree in Computer Science from the University of Kent (Canterbury, England) in 1987. In 1987, he worked as a Consultant for Caplin Cybernetics Corporation (London, England), where he helped in the design of a number of image processing algorithms that were targeted at a particular parallel machine architecture. Prof. Arabnia is currently a Full Professor of Computer Science at University of Georgia, where he has been since October 1987. His research interests include Parallel and distributed processing techniques and algorithms, interconnection networks, and applications. Prof. Arabnia is the founding chair of WORLDCOMP Congress. He is Editor-in-Chief of The Journal of Supercomputing (Springer) and is on the editorial and advisory boards of 17 other journals and magazines. He is the chair of the world committee of PDPTA and is on the Advisory Board of IEEE Technical Committee on Scalable Computing. 